\title{
JACOBI NORMING MESHES
}

\section{FEDERico PiAzZON ANd Marco Vianello}

Abstract. We prove by Bernstein inequality that Gauss-Jacobi(-Lobatto) nodes of suitable order are $L^{\infty}$ norming meshes for algebraic polynomials, in a wide range of Jacobi parameters. A similar result holds for trigonometric polynomials on subintervals of the period, by a nonlinear transformation of such nodes and Videnskii inequality.

Mathematics subject classification (2010): 26D05, 41A10, 42A05, 65T40.

Keywords and phrases: Polynomial inequalities, trigonometric polynomial inequalities, univariate norming meshes, Jacobi polynomials.

\section{REFERENCES}

[1] P. Borwein and T. Erdélyi, Polynomials and Polynomial Inequalities, Springer, New York, 1995.

[2] L. Bos AND M. Vianello, Low cardinality admissible meshes on quadrangles, triangles and disks, Math. Inequal. Appl. 15 (2012), 229-235.

[3] L. Bos AND M. Vianello, Subperiodic trigonometric interpolation and quadrature, Appl. Math. Comput. 218 (2012), 10630-10638.

[4] G. Da Fies And M. Vianello, Trigonometric Gaussian quadrature on subintervals of the period, Electron. Trans. Numer. Anal. 39 (2012), 102-112.

[5] H. Ehlich And K. Zeller, Schwankung von Polynomen zwischen Gitterpunkten, Math. Z. 86 (1964), 41-44.

[6] A. Elbert, A. Laforgia And L. G. Rodonò, On the zeros of Jacobi polynomials, Acta Math. Hungar. 64 (1994), 351-359.

[7] W. GAUTSCHI AND C. Giondano, Luigi Gatteschi's work on asymptotics of special functions and their zeros, Numer. Algorithms 49 (2008), 11-31.

[8] B. GuO AND L. WANG, Jacobi approximations in non-uniformly Jacobi-weighted Sobolev spaces, J. Approx. Theory 128 (2004), 1-41.

[9] A. Kroó, On optimal polynomial meshes, J. Approx. Theory 163 (2011), 1107-1124.

[10] D. Leviatan And J. Sidon, Monotone trigonometric approximation, Mediterr. J. Math. 12 (2015), 877-887.

[11] G. Mastroianni And G. V. Milovanovic, Interpolation processes. Basic theory and applications, Springer Monographs in Mathematics, Springer-Verlag, Berlin, 2008.

[12] F. W. J. Olver, D. W. Lozier, R. F. Boisvert and C. W. Clark, editors, NIST Handbook of Mathematical Functions, Cambridge University Press, New York, 2010.

[13] A. Sommariva And M. Vianello, Polynomial fitting and interpolation on circular sections, Appl. Math. Comput. 258 (2015), 410-424.

[14] G. Szegö, Orthogonal Polynomials, Amer. Math. Soc., Providence, 1975.

[15] M. Vianello, Norming meshes by Bernstein-like inequalities, Math. Inequal. Appl. 17 (2014), 929 936. 